\title{
Absolute proper motions of water masers in NGC 281 measured with VERA
}

\author{
Mayumi Sato, ${ }^{1,2}$ Tomoya Hirota, ${ }^{1}$ Mareki Honma, ${ }^{1}$ \\ Hideyuki Kobayashi ${ }^{1}$ and the VERA Project Team ${ }^{1}$ \\ ${ }^{1}$ VERA Office, National Astronomical Observatory, 2-21-1 Osawa, Tokyo 181-8588 JAPAN \\ email: mayumi.sato@nao.ac.jp \\ ${ }^{2}$ Department of Astronomy, The University of Tokyo, Hongo 7-3-1, Tokyo 113-0033, JAPAN
}

\begin{abstract}
We report on absolute proper-motion measurements of $\mathrm{H}_{2} \mathrm{O}$ maser features in the NGC 281 West molecular cloud, located $\sim 320$ pc above the Galactic plane and associated with an HI loop extending from the Galactic plane. We conducted six-epoch phase-referencing observations of the maser source with VERA (VLBI Exploration of Radio Astrometry) over six months since May 2006. The $\mathrm{H}_{2} \mathrm{O}$ maser features are found to be systematically moving toward the southwest and further away from the Galactic plane with a vertical velocity of $\sim 20$ $30 \mathrm{~km} \mathrm{~s}^{-1}$ at its estimated distance of $2.2-3.5 \mathrm{kpc}$. Our new results provide the most direct evidence that the gas in the NGC 281 region was blown out from the Galactic plane, most likely in a superbubble driven by multiple or sequential supernova explosions in the Galactic plane.
\end{abstract}

Keywords. astrometry, Galaxy: kinematics and dynamics, ISM: bubbles, masers

\section{Introduction and results}

NGC $281\left(\alpha_{2000}=00^{\mathrm{h}} 52^{\mathrm{m}}, \delta_{2000}=+56^{\circ} 34^{\prime}\right.$ or $\left.l=123^{\circ} .07, b=-6 .^{\circ} 31\right)$ is an HII region situated $z \sim 320$ pc above the midplane of the Perseus arm of the Galaxy at a heliocentric distance of $2.9 \mathrm{kpc}$ (Guetter \& Turner 1997), surrounded by a giant molecular cloud complex. Megeath et al. (2002) identified this region on a large-scale HI loop extending from the Galactic plane, and found a 270-pc ring of the clouds of the region, expanding at a velocity of $22 \mathrm{~km} \mathrm{~s}^{-1}$ parallel to the plane. They suggested that the clouds have been formed in a fragmenting superbubble shell. In order to investigate the systemic motion of the region, we have conducted six-epoch phase-referencing observations of an $\mathrm{H}_{2} \mathrm{O}$ maser source in the NGC 281 West cloud with VERA over six months since May 2006.

Figure 1 shows the results of relative and absolute proper-motion measurements of the $\mathrm{H}_{2} \mathrm{O}$ maser features in NGC 281 West. We detected 10 maser features persistent for more than two epochs, with radial velocities $V_{\mathrm{LSR}}$ of -39 to $-22 \mathrm{~km} \mathrm{~s}^{-1}$. These include eight features in C3 and 2 features in C1, corresponding to the VLA observations by Tofani et al. (1995). We first examined the internal motions of the maser features in C3, relative to the brightest maser, feature 4 with $V_{\mathrm{LSR}}=-31.8 \mathrm{~km} \mathrm{~s}^{-1}$, and they showed

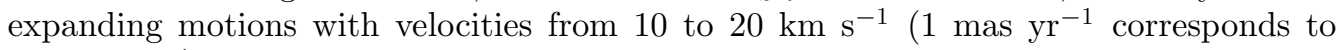
$13.7 \mathrm{~km} \mathrm{~s}^{-1}$ at a distance of $2.9 \mathrm{kpc}$ ). We then measured the absolute proper motions of the maser features 4 (in C3), 9 and 10 (in C1), assuming the distance to NGC 281 to be $2.9 \mathrm{kpc}$ and subtracting the expected parallax from the observed motions. Adopting a different distance of $2.2-3.5 \mathrm{kpc}$ instead of $2.9 \mathrm{kpc}$ in the parallax subtraction yields a difference of $10-15 \%$ in the resultant proper motions. As shown in Figure 1, the mean absolute proper motions of the features in C3 and C1 show a systematic motion toward the southwest. This direction, southwestward, agrees well with that of the proper motion of HD 5005 (the ionizing source of NGC 281), measured with Hipparcos (Perryman et al. 1997). Figure 2 shows a plot of the mean motion of the maser features relative to the 

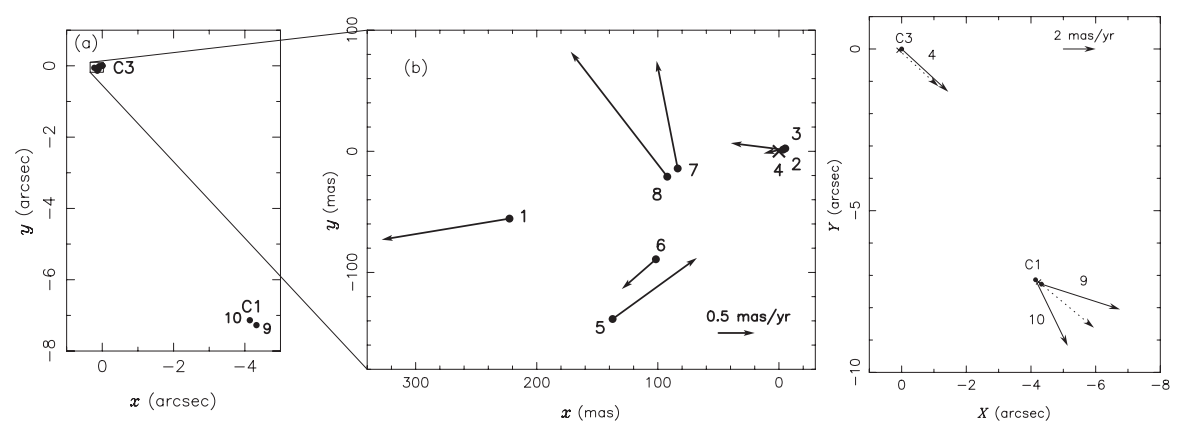

Figure 1. (left)(a) The maser feature distribution; (b) the internal motions of features in C3 relative to feature 4 (denoted by X). (right) A map of the absolute proper motions of features 4 , 9 and 10 (solid arrows) and the mean proper motions in C3 and C1 (dotted arrows). The map origin is set at $\mathrm{RA}(\mathrm{J} 2000.0)=00^{\mathrm{h}} 52^{\mathrm{m}} 24 .^{\mathrm{s}} 70086$ and $\mathrm{DEC}(\mathrm{J} 2000.0)=+56^{\circ} 33^{\prime} 50 . " 5270$. From Sato et al. (2007), (C)2007 Astronomical Society of Japan.

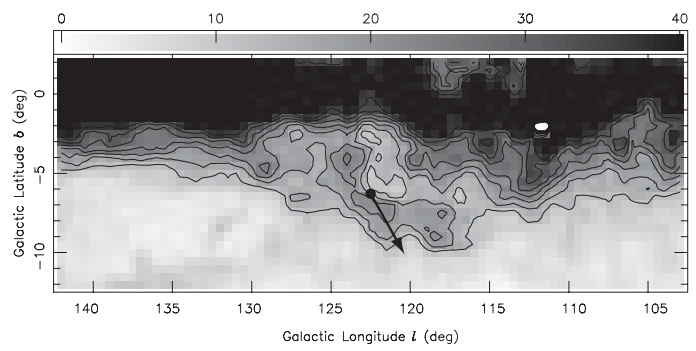

Figure 2. A plot of the mean motion of $\mathrm{H}_{2} \mathrm{O}$ maser features in NGC 281 West relative to the simulated Galactic rotation, superimposed on gray-scale and contour maps of the HI data from Hartmann \& Burton (1997), integrated over $V_{\mathrm{LSR}}=-65$ to $-25 \mathrm{~km} \mathrm{~s}^{-1}$. From Sato et al. (2007), (C)2007 Astronomical Society of Japan.

simulated Galactic rotation, obtained by subtracting the expected apparent motion of NGC 281 due to the Galactic rotation (assumed to be a flat rotation) and the solar motion from the detected proper motion. This mean motion is considered to trace the systemic motion of the region away from the Galactic plane at a transverse velocity of 20$30 \mathrm{~km} \mathrm{~s}^{-1}$ perpendicular to the plane, which is comparable to the ring expansion velocity of $22 \mathrm{~km} \mathrm{~s}^{-1}$ parallel to the plane (Megeath et al. 2002), thus yielding a comparable kinetic energy $\left(\sim 4-8 \times 10^{51}\right.$ ergs $)$ of the clouds for motions perpendicular to the plane as well as parallel. Our measurements provide the most direct evidence that the gas in the NGC 281 region was blown out from the Galactic plane, most likely in a superbubble driven by multiple or sequential supernova explosions in the Galactic plane.

This work is based on Sato et al. (2007) and reproduced with authorization from Astronomical Society of Japan. See Sato et al. (2007) for more details on the measurements and discussions.

\section{References}

Guetter, H. H., \& Turner, D. G. 1997, AJ 113, 2116

Hartmann, D., \& Burton, W. B. 1997, Atlas of Galactic Neutral Hydrogen (Cambridge: Cambridge Univ. Press)

Megeath, S. T., Biller, B., Dame, T. M., Leass, E., Whitaker, R. S., \& Wilson, T. L. 2002, in: P.A. Crowther (ed.), ASP Conf. Ser. 267, Hot Star Workshop III: The Earliest Stages of Massive Star Birth (San Francisco: ASP), p. 257

Perryman, M. A. C., et al. 1997, A\&GA (Letters) 323, L49

Sato, M., Hirota, T., Honma, M., Kobayashi, H. et al. 2007, PASJ 59, No. 4, in press

Tofani, G., Felli, M., Taylor, G. B., \& Hunter, T. R. 1995, A\&AS 112, 299 